\title{
BMJ Open The Screen-ICD trial. Screening for anxiety and cognitive therapy intervention for patients with implanted cardioverter defibrillator (ICD): a randomised controlled trial protocol
}

\author{
Selina Kikkenborg Berg, ${ }^{1,2,3}$ Margrethe Herning ${ }^{4}$ Jesper Hastrup Svendsen, ${ }^{1,5}$ \\ Anne Vinggaard Christensen, ${ }^{1}$ Lau Caspar Thygesen ${ }^{2}$
}

To cite: Berg SK, Herning M, Svendsen JH, et al. The Screen-ICD trial. Screening for anxiety and cognitive therapy intervention for patients with implanted cardioverter defibrillator (ICD): a randomised controlled trial protocol. BMJ Open 2016;6:e013186. doi:10.1136/bmjopen-2016013186

- Prepublication history for this paper is available online. To view these files please visit the journal online (http://dx.doi.org/10.1136/ bmjopen-2016-013186).

Received 24 June 2016 Revised 13 September 2016 Accepted 30 September 2016

CrossMark

For numbered affiliations see end of article.

Correspondence to Dr Selina Kikkenborg Berg; selina@rh.dk

\section{ABSTRACT}

Introduction: Previous research shows that patients with an implanted cardioverter defibrillator (ICD) have a fourfold increased mortality risk when suffering from anxiety compared with ICD patients without anxiety. This research supports the screening of ICD patients for anxiety with the purpose of starting relevant intervention.

Methods and analysis: Screen-ICD consists of 3 parts: (1) screening of all hospitalised and outpatient patients at two university hospitals using the Hospital Anxiety and Depression Scale (HADS), scores $\geq 8$ are invited to participate. (2) Assessment of type of anxiety by Structured Clinical Interview for DSM Disorders (SCID). (3) Investigator-initiated randomised clinical superiority trial with blinded outcome assessment, with 1:1 randomisation to cognitive-behavioural therapy (CBT) performed by a cardiac nurse with CBT training, plus usual care or usual care alone. The primary outcome is HADS-A measured at 16 weeks. Secondary outcomes include Becks Anxiety Inventory, HeartQoL, Hamilton Anxiety Scale, heart rate variability, ICD shock, time to first shock and antitachycardia pacing. A total of 88 participants will be included. The primary analyses are based on the intention-to-treat principle and we use a mixed model with repeated measurements for continuous outcomes. For binary outcomes (HADS-A score $<8$ ), we use a generalised mixed model with repeated measurements.

Ethics and dissemination: The trial is performed in accordance with the Declaration of Helsinki. All patients must give informed consent prior to participation and the trial is initiated after approval by the Danish Data Protection Agency (RH-2015-282) and the regional ethics committee ( $\mathrm{H}-16018868)$. Positive, neutral and negative results of the trial will be published.

Trial registration number: NCT02713360.

\section{INTRODUCTION}

\section{Background and rationale}

Patients with an implanted cardioverter defibrillator (ICD) have a fourfold increased

\section{Strengths and limitations of this study}

- This is the first study to screen for anxiety and diagnose the type of anxiety in implanted cardioverter defibrillator (ICD) patients based on the DSM-IV criteria and test a cognitive-behavioural therapy (CBT) intervention aimed at that specific type of anxiety.

- The interventional personnel include cardiac nurses with a CBT education, making this intervention easy to implement if positive outcomes are found.

- Knowledge from this research is strongly needed as many patients with ICD suffer from anxiety, which is associated with mortality and morbidity.

- The trial is limited by possible non-responders. The screening for anxiety is self-reported and some patients need to mail the screening test back by mail.

mortality risk when suffering from anxiety compared with ICD patients without anxiety, adjusted for age, sex, marital status and comorbidity. This was established in our research from $2014^{1}$ and has led us to recommend anxiety screening of ICD patients ${ }^{2}$ with the purpose of starting relevant therapy.

About $20-25 \%$ of all ICD patients experience anxiety. ${ }^{3}$ The burden does not seem to be different depending on indication. ${ }^{4}$ Data from the Danish Health and Medicines Authority show that anxiety is the one condition that causes the highest amount of lost work hours and costs Danish society around 8.6 billion DKK in lost earnings. ${ }^{5}$

Europe has an average ICD implantation rate of 140 per million inhabitants and in 2013, a total of 1180 first-time ICDs were implanted in Denmark, equalling 214 per million per year. In total, 8000 citizens in Denmark live with an ICD. ${ }^{6}$ 
There are physiological and behavioural processes that lead to inappropriate cardiac outcomes due to anxiety. Suggested models of the relationship between psychology and heart disease highlight the role of the autonomic nervous system. Psychological factors stimulate the autonomic nervous system, which triggers production of catecholamines, increases blood pressure, decreases plasma volume, constricts coronary arteries and increases cardiac demand, platelet activity, coagulation and inflammation. Consequently, patients suffer increased thrombogenesis, arrhythmogenesis, reduced heart rate variability, increased myocardial oxygen demand, myocardial ischaemia and impaired ventricular function. ${ }^{7}$ Moser and Jong have summarised the behavioural mechanisms that are another link between anxiety and cardiac disease. Individuals with high anxiety (compared with non-anxious individuals) have more unhealthy food intake,,$^{8-10}$ smoke more, ${ }^{8-11}$ consume more drugs or alcohol, ${ }^{8}{ }^{10}$ are less compliant to treatment, ${ }^{12}$ sleep poorly ${ }^{8}{ }^{10}$ and are less physically active. $^{8-10}$ All these elements are risk factors associated with progression of cardiac disease. ${ }^{13}$

A review from 2014 concludes that interventions with cognitive-behavioural therapy (CBT) can reduce anxiety in ICD patients. ${ }^{14}$ A total of 11 studies investigated the effect of CBT in ICD patients over a period of 13 years. There were two randomised controlled trials (RCTs) ${ }^{15}{ }^{16}$ one prospective observational study, ${ }^{17}$ one cross-sectional study, ${ }^{18}$ four case-control studies ${ }^{19-22}$ and three case studies. ${ }^{23-25}$ These studies suffer from methodological limitations, which is why conclusive evidence does not exist. However, the two RCTs both find a significant difference in anxiety over time in the CBT group compared with the control group. ${ }^{15}{ }^{19}$ In both trials, the inclusion did not distinguish between patients with and without anxiety and no screening was performed. In a paper from 2015, screening was performed in 690 ICD patients. Moderate to severe anxiety was found in 70 patients and 29 were randomised in a pilot CBT versus usual care trial. Significant differences were found after three CBT sessions. ${ }^{26}$ All interventional trials were small and the intervention was very short. No trial has tested a set-up with screening, identification of type of anxiety using Diagnostic and Statistical Manual of Mental Disorders (DSM) ${ }^{27}$ criteria and a longer lasting intervention, based on current recommendations. ${ }^{28}$

\section{Objectives}

The aim of Screen-ICD is to (1) screen for anxiety, (2) determine the type of anxiety in ICD patients and (3) investigate the effect of a CBT intervention plus usual care compared with usual care alone.

The hypothesis is that there will be a significant difference in anxiety scores between intervention and usual care groups after intervention, in favour of the intervention group.
METHODS

\section{Trial design}

Screen-ICD is an investigator-initiated randomised clinical superiority trial with blinded outcome assessment, with 1:1 randomisation to CBT plus usual care or usual care alone.

\section{Study setting}

Inclusion from two Danish urban university hospital sites.

\section{Eligibility criteria}

Patients eligible for the trial must comply with all of the following at randomisation: score 8 or more in the Hospital Anxiety and Depression Scale-Anxiety (HADS-A), score under 11 on the Hospital Anxiety and Depression Scale-Depression (HADS-D) (unless the HADS-A score exceeds the HADS-D score, anxiety must be the primary problem), be over 18 years of age, have had ICD implantation at any time but at least for 8 weeks, speak and understand Danish and give written informed consent. The Consort flow chart is presented in figure 1 .

\section{Experimental intervention}

The CBT intervention will aim at supporting patients to cope with their heart disease and the limitations and possibilities it brings to life. It will also focus on lifestyle habits. CBT will be targeted on the specific type of anxiety based on the DSM criteria. The therapy will focus on the patient's thoughts and feelings and the actions that come with it, or the fundamental assumptions that cause these thoughts.

The methods of the therapeutic procedure are based on learning theories and the fundament of cognitive therapy was established by the psychiatrist Aron T. Beck in the 1960s. ${ }^{29}$ For each type of anxiety (panic disorder, agoraphobia, social phobia, specific phobia, obsessive phobia, post-traumatic stress, generalised anxiety, anxiety due to a general medical condition, anxiety disorder not otherwise specified), a specific protocol will be followed based on CBT. The treatment of anxiety in ICD patients will contain some overall components:

1. Analysis of the problem: The patient's actual problems will be identified and related to life story relations and components that influence this, for example, cardiac arrest. The way in which assumptions and strategies established earlier in life have an impact on the problem and on the negative thoughts that are characteristic in relation to anxiety will be investigated.

2. Psycho-education: This focuses on the dissemination of information to the patient about the heart disease, ICD and coping with everyday life. In relation to anxiety, there will often be a need to help the patient gain insight into the sympathetic nervous system's role in relation to development of anxiety and increase knowledge of the association between 


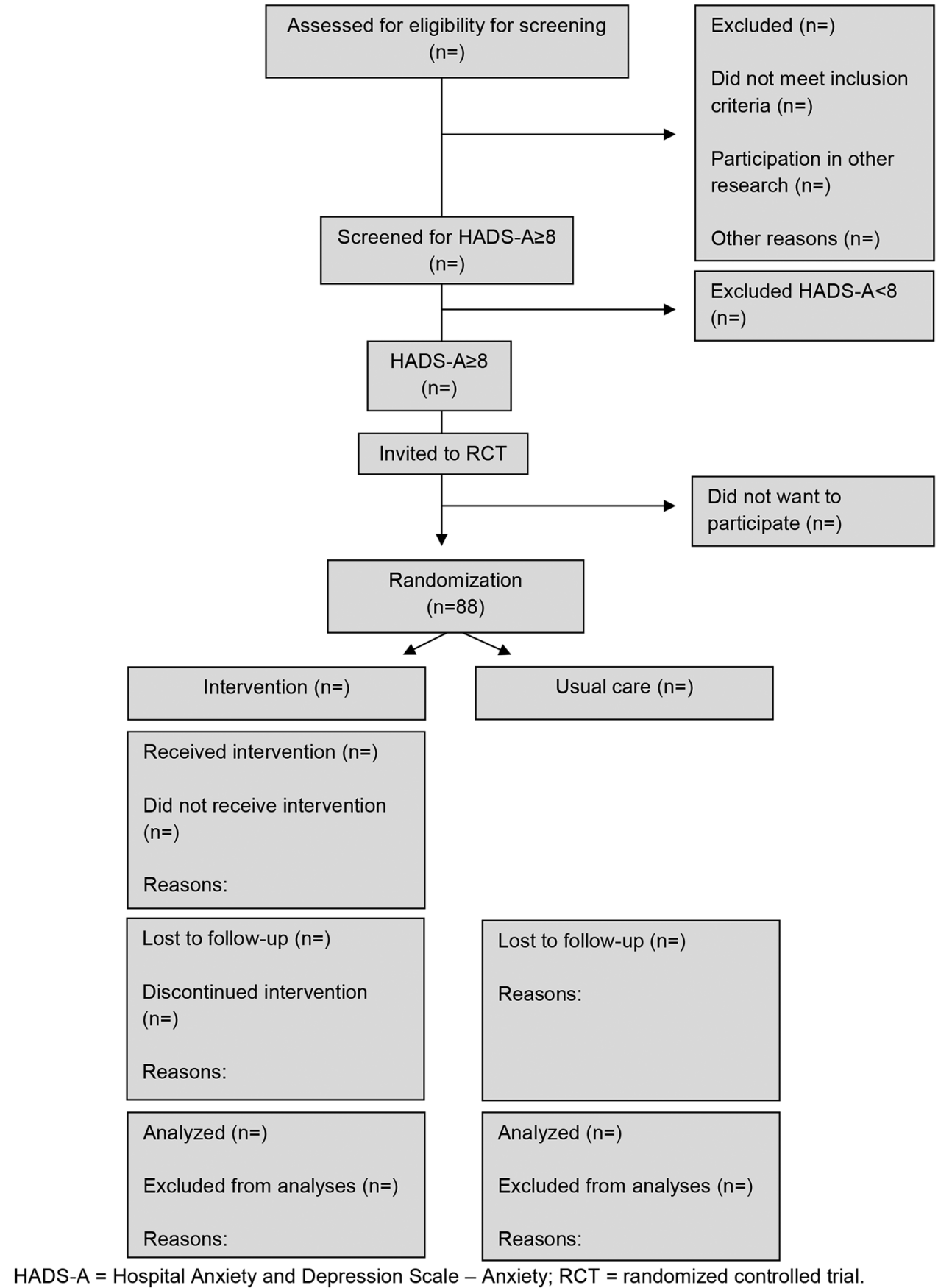

Figure 1 Flow chart.

negative automatic thoughts (catastrophic thoughts), bodily intuitions, feelings and actions and how, for example, behaviour experiments/exposure can reduce anxiety in many situations.

3. Restructuring of negative automatic thoughts: Preparation of a thought journal where the nurse and patients analyse problematic situations by distinguishing 'situation', 'physical feelings', 'feelings', 'automatic negative way of thinking' and 'behaviour'.

4. Planned behaviour experiments, consisting of systematic exposure to situations that trigger the anxiety.
5. Homework, for example, registration of the relationship between thoughts, feelings and bodily sensations. These registrations form the foundation of content in the following therapy: behaviour experiments as practical training or exercises in mastering. ${ }^{29}$

The intervention is considered finalised if the patient has a HADS-A score under 8 after 2 consecutive consultations or after 15 consultations.

Adherence is when the patient participates in all consultations until HADS-A scores are below 8 for 2 consecutive weeks. 
There are no restrictions on concomitant care in the trial period.

\section{Usual care}

Both groups will receive usual care, which consists of relevant medical therapy of the heart disease and standard follow-up of their ICD treatment according to current guidelines. At one of the sites, patients are invited to participate in a 2-hour patient education meeting after ICD implantation, held every second month.

\section{Outcomes}

Demographic variables and clinical characteristics will be collected from patients and from patient records. Data will be registered by trial staff when informed consent is obtained.

Patient screening is performed by patients filling out the self-reported HADS questionnaire.

Included patients will be interviewed with the aim of determining the type of anxiety based on the Structured Clinical Interview for DSM disorders (SCID-1). ${ }^{30} 31$

Patient-reported outcomes, investigator-evaluated outcomes and objective outcomes are included.

The primary outcome of the RCT is anxiety measured by HADS. HADS is a 14-item questionnaire that assesses anxiety and depression level in medically ill persons who are not admitted in psychiatric wards. The scale offers two scores, HADS-A and HADS-D, and consists of seven questions to assess anxiety and seven questions to assess depression. HADS is a validated tool with Cronbach's $\alpha$ of 0.83 and 0.82 . Scores of $0-7$ for the two subscales are regarded as normal and scores of 8-10 suggest the presence of a mood disorder. Scores of 11 and above suggest the probable presence of a mood disorder. ${ }^{32}$

\section{Secondary outcomes}

Several secondary outcomes are planned.

Becks Anxiety Inventory (BAI): A self-reported measure of anxiety with a focus on somatic symptoms of anxiety. It was developed as a measure to discriminate between anxiety and depression. ${ }^{33}$ The 21 -item questionnaire assesses symptoms such as nervousness, dizziness and fear of dying. The BAI score ranges from 0 to 63 and is interpreted as follows: $0-9$, normal or no anxiety; $10-18$, mild to moderate anxiety; 19-29, moderate to severe anxiety; and 30-63, severe anxiety. The BAI has been proved to be highly internally consistent with Cronbach's $\alpha$ of 0.94 and acceptably reliable over an average time lapse of 11 days $(\mathrm{r}=0.67){ }^{34}$

The Hamilton Anxiety Scale (HAM-A): A clinical interview rating scale of the psychic and somatic aspects of anxiety. The scale consists of 14 clinical symptoms that are rated on a 5-point Likert scale ranging from 0 (not present) to 4 (very severe). ${ }^{35}{ }^{36} \mathrm{~A}$ manual for the rating of symptoms is used. The score ranges from 0 to 56 and a score of $0-7$ is interpreted as no anxiety disorder, 8-14 as questionable anxiety disorder, 15-19 as mild anxiety disorder, 20-29 as moderate anxiety disorder and 30-56 as severe anxiety disorder. The reliability and concurrent validity of the HAM-A has proved to be sufficient with an intraclass coefficient of 0.74 and a Spearman coefficient of $0.63 .^{37}$

HeartQoL: A disease-specific questionnaire that measures health-related quality of life in patients with heart disease. The questionnaire consists of 14 items and provides two subscales: a 10-item physical subscale and a 4 -item emotional subscale, which are scored from 0 to $3{ }^{38}$ The questionnaire has proven to be a reliable instrument with Cronbach's $\alpha$ between 0.80 and 0.91 for the global score and each subscale and to be responsive in patients with a wide spectrum of cardiac diagnoses. ${ }^{38-40}$

\section{Exploratory outcomes}

ICD shock, time to first shock and antitachycardia pacing (ATP) will be evaluated due to the risk of arrhythmia stimulated by increased activation of the autonomic nervous system. Data will be gathered from the national ICD register that holds information on all national ICD and cardiac resynchronisation therapy device implantations. No difference is expected between groups due to the small sample size. We will still explore this outcome for the purpose of later meta-analyses and data gathering for sample size estimation in future trials.

Heart rate variability: Evo, Spacelab USA, is a portable device for continuous monitoring of various electrical variables of the cardiovascular system. Heart rate variability, blood pressure and heart rate are all responsive to sympathetic activity, which can be increased by anxiety. Heart rate variability refers to the beat-to-beat variation in the RR interval and is a marker of autonomic nervous system activity. ${ }^{41}$ Reduced heart rate variability is a powerful and independent predictor of short-term and long-term mortality in cardiac patients. ${ }^{42}{ }^{43}$ Higher levels of anxiety are associated with reduced heart rate variability. ${ }^{44}$ Owing to the small sample size, this is not regarded as an outcome, but rather data collection for future sample size calculations in larger trials. Data will only be obtained in the intervention group.

\section{Ancillary questions}

Questions about health-related lifestyle and their consequences: sleep quality, physical activity, alcohol, smoking and weight.

\section{Demographic and clinical baseline information at inclusion}

From patient record: Age, gender, heart disease and comorbidity, ICD indication, type of ICD, prior VT/VF, NYHA-classification, ejection fraction, diabetes mellitus and all medication.

Measurements: Heart rate variability (intervention group only), weight, pulse, blood pressure. 
Demographic and clinical information after intervention

(at 16 weeks)

From questionnaire: All medication, other treatment for anxiety in the project period, hospital admissions in the project period.

Measurements: Heart rate variability (intervention group only), weight, pulse, blood pressure.

\section{Participant timeline}

The timeline is presented in figure 2 .

\section{Sample size and power estimations}

Sample size

A score below 8 on HADS-A is regarded as a normal score. We wish to see a significant difference between the intervention and control groups to be sure that development over time is not caused by a spontaneous recovery but is an intervention effect.

An earlier study ( $\mathrm{n}=334)$ showed that patients scoring more than 8 on HADS-A had a mean score of 10.2 with an SD of 2.3. ${ }^{1}$ If the patients with anxiety have a score below 8 , then the true difference between the intervention and control groups should be 2.3 points.

With a power of $90 \%$ and a type 1 error set at $0.05,22$ patients should be entered in each group. There could be a spontaneous improvement in the control group and not all patients may benefit from CBT. Furthermore, we expect withdrawal from the study; this is typically around $20-30 \%$ in similar intervention studies. As a result, we double the inclusion uptake to strengthen the trial, which means that we will need 44 intervention participants and 44 control participants (88 participants in total)

\section{Power estimations for secondary outcomes}

BAI: In a previous study, the response within each participant group was normally distributed with an SD of $5 .{ }^{45}$ If the true difference between the experimental and control means is 5.2 , we will be able to reject the null hypothesis that the population means of the experimental and control groups are equal with a probability (power) of 0.998 .

HAM-A: Based on previous studies, the SD was set at $7{ }^{45-47}$ If the true difference in the experimental and control means is 7 , we will be able to reject the null hypothesis that the population means of the experimental and control groups are equal with a probability (power) of 0.996 .

HeartQoL: In a previous study, the response within each participant group was normally distributed with an $\mathrm{SD}$ of $0.75 .^{1}$ If the true difference in the experimental and control means is 0.4 , we will be able to reject the null hypothesis that the population means of the experimental and control groups are equal with a probability (power) of 0.696 .

\section{Recruitment}

All patients with ICD aged over 18 years who are admitted to the hospital or at outpatient visit at one of the two sites doing the time period of the trial are invited to fill out a questionnaire (HADS) screening for anxiety. By screening patients at all types of hospital contacts, patients with newly implanted ICDs and patients who have had an ICD for many years will be included. In patients who received a shock or were admitted to the hospital, screening will take place 8 weeks after the event to allow for normal restitution. The screening will continue until the target sample size is archived.

If a patient, after receiving verbal and written information, decides to participate in the Screen-ICD trial, an informed consent form will be signed and they will be randomised to either: (1) a CBT intervention and usual care or (2) usual care alone. The intervention is performed by cardiac nurses with a certified cognitive therapy education. The nurses are supervised by a psychologist.

\section{Allocation}

Participants will be randomised 1:1 to the experimental intervention group or to the control group. Randomisation will be conducted using the web-based tool Randomizer for Clinical Trials. The allocation sequence will be computer-generated with a block size of 4 , concealed from the investigators. The allocation will be conducted when the investigator calls a voice respondent who logs in to 'Randomizer for Clinical Trials 1.8.1', selects relevant participant information (participant number and stratum) and assigns the participant to either intervention or control by phone to the investigator. The strata are severity of anxiety measured by HADS-A (8-10 or 11-21) and age $(\leq 65$ or $>65$ years $)$.

\section{Blinding}

Owing to the conditions required for psycho-educational interventions, it is not possible to blind the intervention staff and patients. All baseline information and clinical interviews are performed before randomisation. Data management and administration will be performed by blinded staff. Statistical analysis of outcomes and conclusions from these will be blinded. Results of the trial are analysed by an independent statistician, and the results will be interpreted by the research group. The conclusion will be prepared in two versions, before the allocation code is broken, with the two arms alternatively assumed as intervention (one that assumes that arm A is the intervention, and second, that assumes that the arm $\mathrm{B}$ is intervention).

\section{Data collection}

The questionnaires for screening and collection of secondary outcomes are self-administered paper questionnaires handed out to the patient at the hospital or sent to their home address. 
Figure 2 Participant timeline.

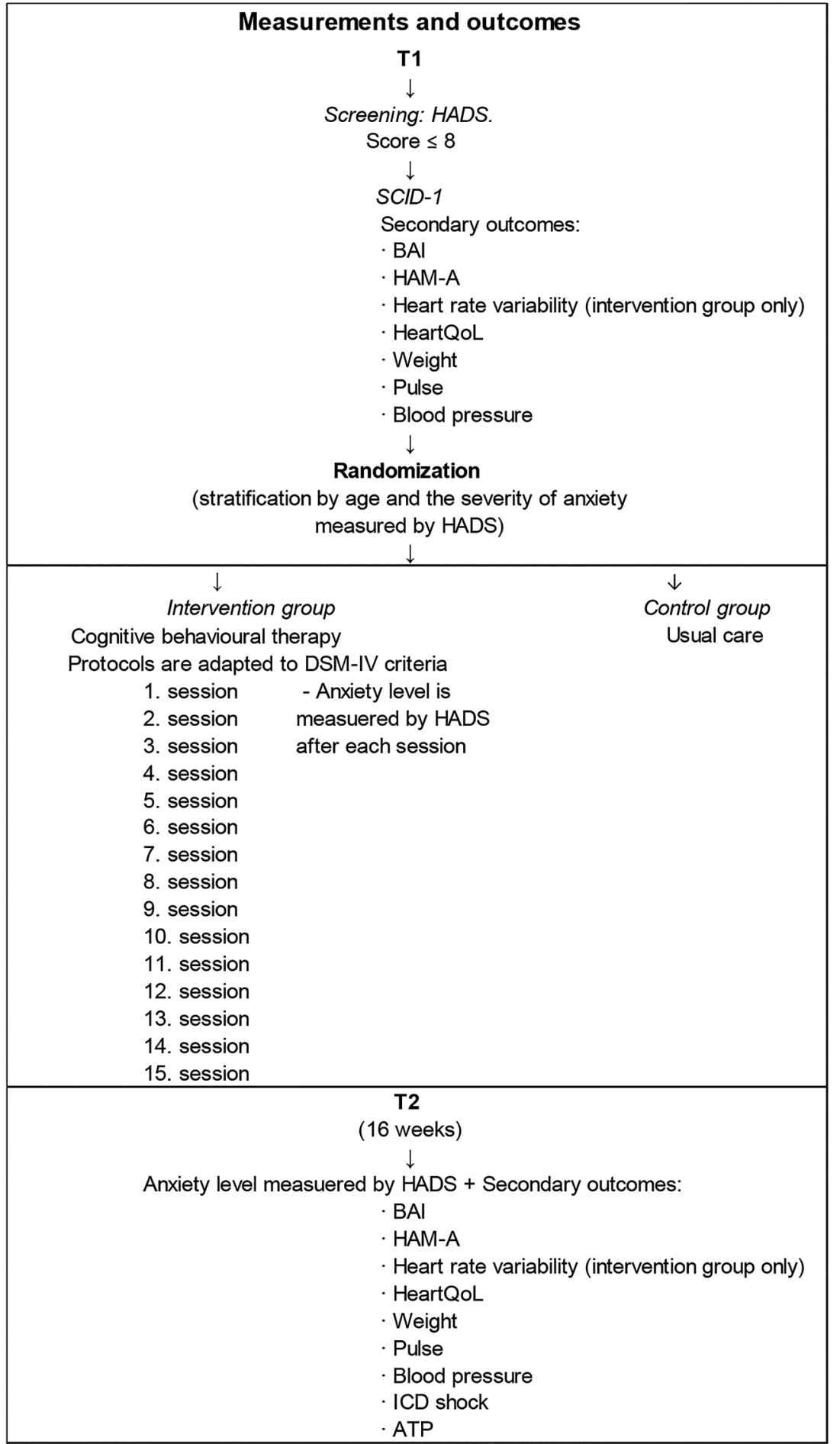

The SCID-1 and HAM-A interviews are performed by five trained cardiac nurses. The first three interviews are performed by two nurses together to allow for training and assure inter-rater concordance.

Trial participants are free to withdraw their informed consent at any time and be treated according to the department's standard procedures. Patients who leave the trial will be asked for permission to continue to collect data and to use already collected data. If the patient gives permission, data will be included in the final analysis.

\section{Data management}

Questionnaire responses will be typed and stored in a secure folder with limited access on the hospital server. The questionnaire data will be placed in one folder and identified with a study participant number and in another folder, the participant's personal identification number will be stored with the matching study participant number.

Individual patient data will be handled as normal data and records will be protected according to the Act on Processing of Personal Data and the Danish Health Care 
Act. Data will be stored in accordance with Danish Data Protection Agency rules. Anonymous data that are encoded with the individual patient code will be entered into the computerised database and transferred for analysis portal in encrypted mode. This system meets all criteria for the handling of patient data in accordance with the laws on the processing of personal data. All original records are stored at Rigshospitalet for 15 years to allow inspections by competent authorities. The trial database will be preserved for 15 years and anonymised. After analysis, experimental data will be submitted to the Danish Data Archives.

\section{Statistical analysis}

The primary analyses will be performed according to the intention-to-treat principle and we will use a mixed model with repeated measurements for continuous outcomes. For binary outcomes (HADS-A score $<8$ ), we use a generalised mixed model with repeated measurements. By using mixed models, we ensure that missing data do not create bias as long as they are missing at random. In the case of significant results in the primary outcome, sensitivity analyses will be performed to estimate the potential effect of data missing at random by a worst-case scenario. Let A be the group where a beneficial significant effect is observed and B be the other group. Missing values in group A will be imputed by the minimum value found in the material and missing values in group B will be imputed by the maximum value found. The primary outcome will first be tested using a significance level of 0.05 . Analyses of the secondary outcome measures as preplanned in the protocol will be analysed with no adjustment of $\mathrm{p}$ values due to multiplicity. Instead, the interpretation of each secondary outcome measure will be assessed in the light of multiple testing, that is, statistically significant effects will be interpreted in the context of increased risk of type I error. No significance testing will be performed for the exploratory outcomes. The clinical effect size is analysed by Cohen's d. Significance level is set at 5\%. SAS V.9.3 is used for the analyses (SAS Institute, Cary, North Carolina, USA).

\section{Data monitoring}

Owing to the small sample size, no data monitoring committee is established and no interim analyses will be performed.

\section{Harms}

No risks are expected. CBT is a safe, non-invasive, nonpharmacological treatment. There is a potential beneficial effect of participation in CBT as anxiety levels may decrease. ${ }^{14}$ To avoid interference with normal restitution, an 8-week time span after an event must be upheld before screening for anxiety. Adverse events will be continuously monitored.

\section{Ethics}

The trial is performed in accordance with the Declaration of Helsinki in its latest form. All patients must give informed consent to the investigators prior to participation. All study- related information will be stored securely at the study site. All informed consent forms signed by participants will be stored in locked file cabinets in areas with limited access. The trial is initiated after approval by the Danish Data Protection Agency (RH-2015-282) and the regional ethics committee (H-16018868). The regional ethics committee will be asked for permission in the case of protocol amendments. The trial is registered at http://www. ClinicalTrials.gov (NCT02713360). All investigators will be given full access to the final trial data set.

\section{Dissemination}

Positive, neutral and negative results of the trial will be published. The final manuscripts originating from the trial will be sent to a peer-reviewed international journal. Authorship will be allocated using the guidelines for authorship set out by the International Committee of Medical Journal Editors and will depend on the personal involvement of each author.

\section{TRAJECTORY}

The trial will begin in May 2016 with inclusion of the first participants. Inclusion will end when 88 patients are enrolled in the RCT, expected to be by the end of 2016 . Data collection will end by mid-2017.

\section{Author affiliations}

${ }^{1}$ The Heart Centre, Rigshospitalet, Copenhagen University Hospital, Copenhagen, Denmark

${ }^{2}$ National Institute of Public Health, University of Southern Denmark, Copenhagen, Denmark

${ }^{3}$ Department of Public Health, Faculty of Health and Medical Sciences, University of Copenhagen, Copenhagen, Denmark

${ }^{4}$ Department of Cardiology, Herlev and Gentofte University Hospital, Hellerup, Denmark

${ }^{5}$ Department of Clinical Medicine, Faculty of Health and Medical Sciences, University of Copenhagen, Copenhagen, Denmark

Acknowledgements To Mette Kirstine Wagner, Kim Mechta Nielsen, Trine Bernholdt Rasmussen, Lene Høgh Simonsen Søderberg, Rikke Brandt, Camilla Dichman, Maria Sofie Qvist Hoelgaard, Pia Callesen, Martin Balslev Jørgensen, Carsten Juul, Frida Richter Hansen, Frederikke Stage Kristensen and the Screen-ICD advisory group for their invaluable contribution to the preparation of the trial.

Contributors SKB conceived the idea of the study. SKB, MH, JHS and AVC initiated the study design and implementation. LCT and SKB developed the plan for the statistical analyses. All contributed to the refinement of the study protocol.

Funding Screen-ICD will be conducted at the Heart Centre, Rigshospitalet and at the Department of Cardiology at Herlev and Gentofte Hospital. The trial is funded by the above sites and by Trygfonden ID 117364.

Competing interests None declared.

Ethics approval The regional ethics committee (H-16018868).

Provenance and peer review Not commissioned; externally peer reviewed.

Open Access This is an Open Access article distributed in accordance with the Creative Commons Attribution Non Commercial (CC BY-NC 4.0) license, which permits others to distribute, remix, adapt, build upon this work noncommercially, and license their derivative works on different terms, provided 
the original work is properly cited and the use is non-commercial. See: http:// creativecommons.org/licenses/by-nc/4.0/

\section{REFERENCES}

1. Berg SK, Thygesen LC, Svendsen JH, et al. Anxiety predicts mortality in ICD patients: results from the cross-sectional National Copenheart Survey with Register follow-up. Pacing Clin Electrophysiol 2014;37:1641-50.

2. Pedersen FP. Angst kan koste hjertesyge livet. Berlingske, 2014.

3. Dunbar SB, Dougherty CM, Sears SF, et al. Educational and psychological interventions to improve outcomes for recipients of implantable cardioverter defibrillators and their families: a scientific statement from the American Heart Association. Circulation 2012;126:2146-72.

4. Pedersen SS, Sears SF, Burg MM, et al. Does ICD indication affect quality of life and levels of distress? Pacing Clin Electrophysiol 2009;32:153-6.

5. Sundhedsstyrelsen. Sygdomsbyrden i Danmark. Sundhedsstyrelsen, 2015.

6. Sundhedsstyrelsen. Pacemakere, ICD'er og andre avancerede pacemakersystemer. Sundhedsstyrelsen, 2014.

7. Molinari E, Compare A, Parati G. Clinical psychology and heart disease. Italy: Springer, 2006.

8. Buselli EF, Stuart EM. Influence of psychosocial factors and biopsychosocial interventions on outcomes after myocardial infarction. J Cardiovasc Nurs 1999;13:60-72.

9. Hayward C. Psychiatric illness and cardiovascular disease risk Epidemiol Rev 1995;17:129-38.

10. Sirois BC, Burg MM. Negative emotion and coronary heart disease. A review. Behav Modif 2003;27:83-102.

11. Kubzansky LD, Kawachi I, Weiss ST, et al. Anxiety and coronary heart disease: a synthesis of epidemiological, psychological, and experimental evidence. Ann Behav Med 1998;20:47-58.

12. Frasure-Smith $N$, Lesperance $F$, Talajic $M$. The impact of negative emotions on prognosis following myocardial infarction: is it more than depression? Health Psychol 1995;14:388-98.

13. Molinari E, Compare A, Parati G, eds. Clinical Psychology and Heart Disease. Cap 8. Moser DK, DeJong MJ. Springer, 2006.

14. Maia AC, Braga AA, Soares-Filho G, et al. Efficacy of cognitive behavioral therapy in reducing psychiatric symptoms in patients with implantable cardioverter defibrillator: an integrative review. Braz $J$ Med Biol Res 2014:47:265-72.

15. Chevalier P, Cottraux J, Mollard E, et al. Prevention of implantable defibrillator shocks by cognitive behavioral therapy: a pilot trial. $A m$ Heart J 2006;151:191.

16. Irvine J, Stanley J, Ong L, et al. Acceptability of a cognitive behavior therapy intervention to implantable cardioverter defibrillator recipients. J Cogn Psychother 2010;24:246-64.

17. Kohn CS, Petrucci RJ, Baessler C, et al. The effect of psychological intervention on patients' long-term adjustment to the ICD: a prospective study. Pacing Clin Electrophysiol 2000;23:450-6.

18. Steinke EE. Intimacy needs and chronic illness: strategies for sexual counseling and self-management. J Gerontol Nurs 2005;31:40-50.

19. Irvine J, Firestone J, Ong L, et al. A randomized controlled trial of cognitive behavior therapy tailored to psychological adaptation to an implantable cardioverter defibrillator. Psychosom Med 2011;73:226-33.

20. Lewin RJ, Coulton S, Frizelle DJ, et al. A brief cognitive behavioral preimplantation and rehabilitation programme for patients receiving an implantable cardioverter-defibrillator improves physical health and reduces psychological morbidity and unplanned readmissions. Heart 2009;95:63-9.

21. Frizelle DJ, Lewin RJ, Kaye G, et al. Cognitive-behavioral rehabilitation programme for patients with an implanted cardioverter defibrillator: a pilot study. Br J Health Psychol 2004;9:381-92.

22. Vazquez LD, Conti JB, Sears SF. Female-specific education, management, and lifestyle enhancement for implantable cardioverter defibrillator patients: the FEMALE-ICD study. Pacing Clin Electrophysiol 2010;33:1131-40.

23. Smith LC, Fogel D, Friedman S. Cognitive-behavioral treatment of panic disorder with agoraphobia triggered by AICD implant activity Psychosomatics 1998;39:474-7.

24. Hirsh AT, Sears SF Jr, Conti JB. Cognitive and behavioral treatments for anxiety and depression in a patient with an implantable cardioverter defibrillator (ICD): a case report and clinical discussion. J Clin Psychol Med Settings 2009;16:270-9.

25. Sears SF Jr, Conti JB, Curtis AB, et al. Affective distress and implantable cardioverter defibrillators: cases for psychological and behavioral interventions. Pacing Clin Electrophysiol 1999;22:1831-4.

26. Qintar M, George JJ, Panko M, et al. A prospective study of anxiety in ICD patients with a pilot randomized controlled trial of cognitive behavioral therapy for patients with moderate to severe anxiety. J Interv Card Electrophysiol 2015;43:65-75.

27. American Psychiatric Association. DSM-5 diagnostic and statistical manual of mental disorders. 5th edn. Washington, DC: American Psychiatric Association, 2013.

28. Clark DA, Beck AT. Cognitive therapy of anxiety disorders. Science and practice. New York: The Guilford Press, 2010.

29. Arendt M, Rosenberg NK. Kognitiv terapi-Nyeste udvikling. Hans Reitzels Forlag, 2012.

30. Spitzer RL, Williams JB, Gibbon M, et al. The Structured Clinical Interview for DSM-III-R (SCID). I: history, rationale, and description. Arch Gen Psychiatry 1992;49:624-9.

31. Williams JB, Gibbon M, First MB, et al. The Structured Clinical Interview for DSM-III-R (SCID). II. Multisite test-retest reliability. Arch Gen Psychiatry 1992;49:630-6.

32. Bjelland I, Dahl AA, Haug TT, et al. The validity of the Hospital Anxiety and Depression Scale. An updated literature review. $J$ Psychosom Res 2002:52:69-77.

33. Beck AT, Epstein N, Brown G, et al. An inventory for measuring clinical anxiety: psychometric properties. J Consult Clin Psychol 1988:56:893-7.

34. Fydrich T, Dowdall D, Chambless DL. Reliability and validity of the Beck Anxiety Inventory. J Anxiety Disord 1992;6:55-61.

35. Hamilton M. The assessment of anxiety states by rating. $\mathrm{Br} \mathrm{J} \mathrm{Med}$ Psychol 1959;32:50-5.

36. Frank-Stromborg M. Instruments for clinical nursing research Boston, MA: Jones and Bartlett Publishers, 1992.

37. Maier W, Buller R, Philipp M, et al. The Hamilton Anxiety Scale: reliability, validity and sensitivity to change in anxiety and depressive disorders. J Affect Disord 1988;14:61-8.

38. Oldridge N, Hofer S, McGee H, et al. The HeartQoL: Part I. Development of a new core health-related quality of life questionnaire for patients with ischemic heart disease. Eur J Prev Cardiol 2014:21:90-7.

39. Oldridge N, Hofer S, McGee H, et al. The HeartQoL: Part II. Validation of a new core health-related quality of life questionnaire for patients with ischemic heart disease. Eur J Prev Cardiol 2014;21:98-106.

40. Oldridge N, Saner H, McGee HM, et al. The Euro Cardio-QoL Project. An international study to develop a core heart disease health-related quality of life questionnaire, the HeartQoL. Eur J Cardiovasc Prev Rehabil 2005;12:87-94.

41. Heart rate variability: standards of measurement, physiological interpretation and clinical use. Task Force of the European Society of Cardiology and the North American Society of Pacing and Electrophysiology. Circulation 1996;93:1043-65.

42. Stein PK, Domitrovich PP, Huikuri HV, et al. Traditional and nonlinear heart rate variability are each independently associated with mortality after myocardial infarction. $J$ Cardiovasc Electrophysio 2005;16:13-20.

43. Aronson D, Mittleman M, Burger A. Measures of heart period variability as predictors of mortality in hospitalized patients with decompensated congestive heart failure. Am J Cardiol 2004;93:59-63.

44. Carney RM, Freedland KE, Stein PK. Anxiety, depression, and heart rate variability. Psychosom Med 2000;62:84-7.

45. Kim YW, Lee SH, Choi TK, et al. Effectiveness of mindfulness-based cognitive therapy as an adjuvant to pharmacotherapy in patients with panic disorder or generalized anxiety disorder. Depress Anxiety 2009; 26:601-6.

46. van Beek MH, Oude Voshaar RC, Beek AM, et al. A brief cognitive-behavioral intervention for treating depression and panic disorder in patients with noncardiac chest pain: a 24-week randomized controlled trial. Depress Anxiety 2013;30:670-8.

47. Gaudlitz K, Plag J, Dimeo F, et al. Aerobic exercise training facilitates the effectiveness of cognitive behavioral therapy in panic disorder. Depress Anxiety 2015;32:221-8. 